\title{
RTN-based defect tracking technique: experimentally probing the spatial and energy profile of the critical filament region and its correlation with $\mathrm{HfO}_{2}$ RRAM switching operation and failure mechanism
}

\author{
Z. Chai ${ }^{(1)}$, J. Ma ${ }^{(1)}$, W. Zhang ${ }^{(1)}$, B. Govoreanu ${ }^{(2)}$, E. Simoen ${ }^{(2)}$, J. F. Zhang ${ }^{(1)}$, Z. Ji ${ }^{(1)}$, R. Gao ${ }^{(1)}$, G. Groeseneken $^{(2)}$, M. Jurczak ${ }^{(2)}$ \\ ${ }^{(1)}$ Dept. of Electronics \& Electr. Eng., Liverpool John Moores University, Liverpool L3 3AF, UK. ${ }^{(2)}$ IMEC, Leuven B3001, Belgium
}

Introduction: RRAMs are strong candidates for new generations memory technology [1-3]. Filament rupture/restoration induced by movement of defects, e.g. oxygen ions/vacancies $(\mathrm{Vo})$, is considered the switching mechanism in $\mathrm{HfO}_{2}$ RRAM [2-3]. However, details of filament alteration during switching are still speculative, due to lack of experiment-based probing techniques to directly monitor its spatial and energy profiles and to correlate them with the switching/failure mechanism, impeding its understanding and modeling. In this work, an RTN-based defect tracking technique (RDT) is developed to monitor the defect movements and the spatial and energy $\left(X_{T}, E_{T}\right)$ profile of the critical filament region (CFR) for the first time. CFR alterations can be directly correlated with switching operations, and new endurance failure mechanism has been revealed.

Problems: There are currently two types of techniques for providing the information on filament profile. One is AFM/TEM [45], which provides a spatial image of the filament but the technique is destructive, time consuming, and statistics-unfriendly; The other is based on the current measured against bias/time and the filament is modelled via simulation $[3,6]$, in which the defect $\mathrm{X}_{\mathrm{T}} / \mathrm{E}_{\mathrm{T}}$ profiles need to be fitted due to lack of direct experimental results; Hence it is important to provide direct experimental evidence at defect level to investigate the details of filament alteration and link it to switching and failure mechanisms, which is the key advance of this work.

RTN in RRAM: Defect's $\mathrm{X}_{\mathrm{T}} / \mathrm{E}_{\mathrm{T}}$ have been extracted from RTN in MOSFETs based on eq.1\&2 (Fig.1a\&b) [7-8]. RTN in RRAM have also been analyzed [6,9], but no defect $\mathrm{X}_{\mathrm{T}} / \mathrm{E}_{\mathrm{T}}$ were obtained because eq.1\&2 are not applicable in the following cases: 1) defect movement (Vo) and electron trapping/detrapping (e-RTN) can coexist and lead to complex RTN (Fig.3a); 2) e-RTN can also result from electron tunneling through the defect to the opposite electrode or to other defects (Fig.2a) [8]; 3) the large quantity of defects near each electrode leads to metallic-like local conduction (Fig.2b).

To overcome these problems, in this work, we only consider RTNs that clearly follow eq.1\&2, as shown in Fig.1a\&b: 1) all RTNs are then evaluated individually for e-RTN or Vo-movement using the technique described in the next section (Fig.3a-c); 2) This can also exclude the RTNs caused by electrons tunneling through the defects where $\tau_{\mathrm{c}}$ and $\tau_{\mathrm{e}}$ have the same polarity of bias dependence (Fig.2a); 3) Metallic conduction regions are considered as part of the electrodes, and the relative defect spatial location within the electron tunneling conduction region (TCR), $\mathrm{Xt} / \mathrm{T}_{\mathrm{ox}, \mathrm{TCR}}$, (Fig.2b) can be obtained using eq.1. We will show that clear correlations between RTN, defect and filament can be revealed after taking the above into account.

Vo movement tracking by e-RTN: RTN is measured from the $\mathrm{TiN} / \mathrm{Hf} / \mathrm{HfO}_{2} / \mathrm{TiN}$ RRAM cells [2]. Device size is $40 \mathrm{~nm} \times 40 \mathrm{~nm}$, $\mathrm{HfO}_{2}$ thickness is either $3 \mathrm{~nm}$ or $5 \mathrm{~nm}$. Fig.3a illustrates the typical RDT test procedure. When current jumps are observed (Vo) at HRS, RRAM resistance (R@0.1V) and $\mathrm{X}_{\mathrm{T}} / \mathrm{E}_{\mathrm{T}}$ extracted from e-RTNs also change simultaneously (Fig.3a-c), supporting that the current jumps are caused by Vo-movement induced filament alteration. Moreover, both $\mathrm{X}_{\mathrm{T}} / \mathrm{E}_{\mathrm{T}}$ and $\mathrm{R}$ retain their altered values when being re-measured across the bias range, and the defect at the previous location A can no longer be detected (Fig.4), as it has moved away to location B and B'.
To further demonstrate the correlation between defect movement and filament alteration, amplitude of $\Delta \mathrm{R} / \mathrm{R}$ disturbs are analyzed against $X_{T}$ and $E_{T}$ before and after Vo movements. Fig.5a shows that decrease of $R$ at positive bias are correlated with Vo moving towards the highlighted critical filament region (CFR), and in Fig.5b, increase of $\mathrm{R}$ at negative bias are correlated with Vo moving away from CFR. This can be seen clearly in Fig.6a\&b. The largest Iread increases (jump-up) are associated with Vo moving towards CFR $\left(2^{\text {nd }}\right.$ quadrant in Fig.6a). In contrast, the largest Iread decreases (jump-down) are caused by Vo moving out of CFR (4 ${ }^{\text {th }}$ quadrant in Fig.6b). Furthermore, direction of Vo movement is also closely correlated with bias polarity. Current increase at $\mathrm{V}>0.2 \mathrm{~V}$ is associated with $\mathrm{Vo}$ moving towards $\mathrm{BE}$, and current decrease at $\mathrm{V}<-0.2 \mathrm{~V}$ is associated with Vo moving towards TE (Fig.7). At a weaker Eox when $-0.2 \mathrm{~V}<$ $\mathrm{V}<0.2 \mathrm{~V}$, Vo can move in either directions.

Filament alteration: The above technique can be used to monitor filament alteration during switching operations. Defects are extracted during normal switching on/off cycles. The profile of total defects extracted at HRS during the cycling (Fig.8) clearly shows a region near $\mathrm{BE}$ with the least defects, agreeing with the CFR in Fig.3-6. This is also supported by thermal simulation in Ref.10. CFR is modulated by different operation conditions. It is widened at a higher Vreset, leading to a higher R at HRS (Fig.9). Defects exhibit a wider energy distribution at a lower compliance ICC (Fig.10) probably due to weaker "regulation power" during SET. At a LRS where TCR still dominates [11], more defects in the CFR are detected (Fig.11), supporting that CFR determines the device resistance state. Fig.12 shows that CFR is also observed near BE in devices with $3 \mathrm{~nm} \mathrm{HfO}_{2}$.

Failure mechanism: A typical endurance test result is shown in Fig.13, in which the cycling underwent four phases: stable, unstable, and stuck at HRS failure which can then be recovered by applying a higher $\mathrm{V}_{\text {recovery }}$ [2]. After the failure (Fig.14a), CFR at around ET=EF is surrounded by defects at lower/higher energy levels. This reveals the failure mechanism: As defects surrounding CFR reach a critical level, they will (i) repel other defects from moving into CFR, causing SET failure; (ii) may also form a shunt current path at Vset, reducing the bias across CFR and assisting the failure; (iii) hardly contribute to the conduction at Vread due to their energy level misalignment with electrodes and/or longer electron tunneling path. At unstable phase, defects can either move around or into CFR, causing unreliable SET. Moreover, these surrounding defects are removed after the recovery (Fig.14b), strongly supporting their roles in causing failure. Similar failure mode has also been observed under AC operations (Fig.15).

Conclusions: For the first time, an RTN based defect tracking technique has been developed that can monitor the defect movement and filament alteration in RRAM devices. Critical filament region has been identified during switching operation at various conditions and new endurance failure mechanism is revealed. This technique provides a useful tool for RRAM technology development. Reference: [1] Wong et al, IEEE proc., 2012. [2] Govoreanu et al, IEDM, 2011. [3] R. Degraeve et al, VLSI, 2012. [4] Celano, et al, IEDM, 2013. [5] Kwon, et al, IRPS, 2014. [6] Ambrogio et al, IEDM 2013 [7] Chang et al, IEDM 2008. [8] Kirton et al, Advances in Physics, 1989. [9] Raghavan et al, VLSI 2013. [10] Govoreanu et al, IEEE TED, 2013. [11] Wei et al, IEDM, 2015 Acknowledgement: EPSRC of UK (Grant nos.: EP/M006727/1 and EP/L010607/1) 

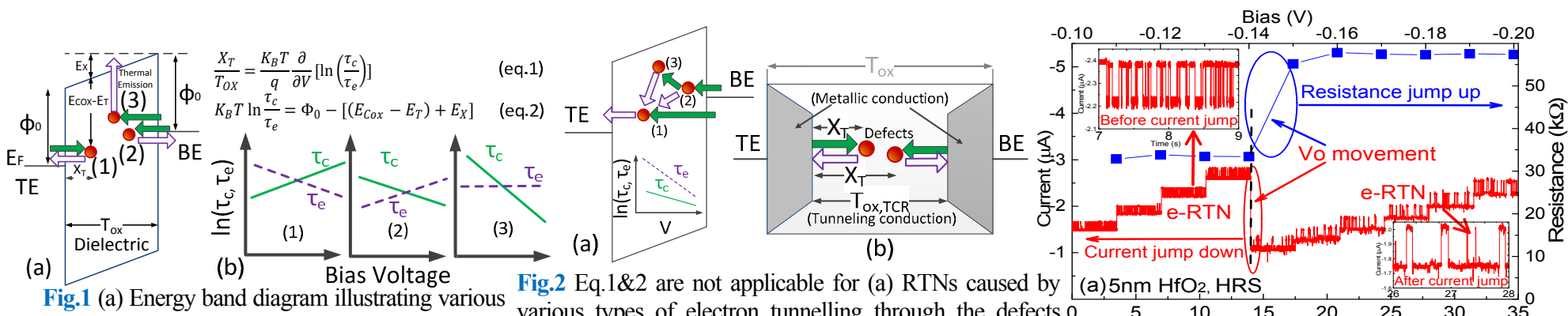

Fig.1 (a) Energy band diagram illustrating various RTNs examined in this work caused by electron tapping and detrapping. (b) Defect's spatial and energy location $\left(\mathrm{X}_{\mathrm{T}}, \mathrm{E}_{\mathrm{T}}\right)$ can be extracted from the capture and emission time constant dependence on the bias for these RTNs by using eq. $1 \& 2[7,8]$.
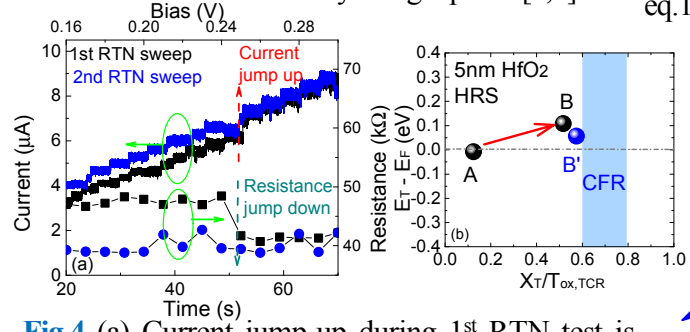

various types of electron tunnelling through the defects where $\tau_{\mathrm{c}}$ and $\tau_{\mathrm{e}}$ have the same polarity of bias dependence; and (b) in metallic conduction region near both electrodes. Defect's relative location within the electron tunnelling conduction region TCR, $\mathrm{X}_{\mathrm{T}} / \mathrm{T}_{\mathrm{Ox}-\mathrm{TCR}}$, can be extracted from eq. 1 even though the absolute value of $\mathrm{T}_{\mathrm{ox}, \mathrm{TCR}}$ is unknown.
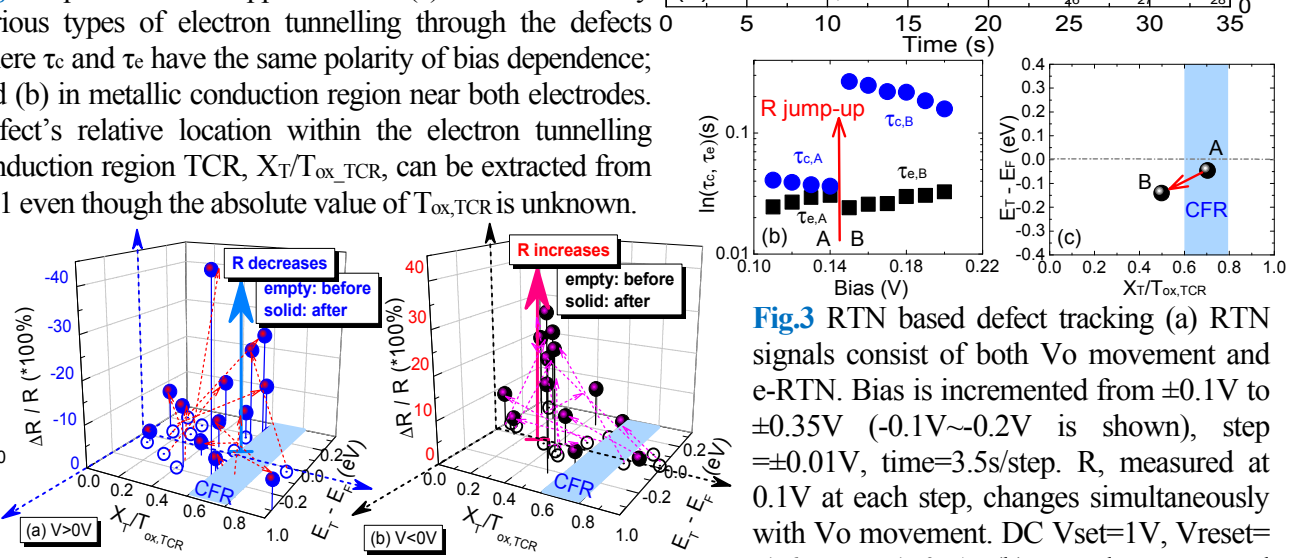

Fig.3 RTN based defect tracking (a) RTN signals consist of both Vo movement and e-RTN. Bias is incremented from $\pm 0.1 \mathrm{~V}$ to $\pm 0.35 \mathrm{~V} \quad(-0.1 \mathrm{~V} \sim-0.2 \mathrm{~V}$ is shown), step $= \pm 0.01 \mathrm{~V}$, time $=3.5 \mathrm{~s} / \mathrm{step} . \mathrm{R}$, measured at $0.1 \mathrm{~V}$ at each step, changes simultaneously with Vo movement. DC Vset $=1 \mathrm{~V}$, Vreset= associated with R jump-down. Both current and R Fig.5 (a) Decreases of R at positive bias are correlated with Vo retain the altered values during $2^{\text {nd }}$ RTN test, moving towards CFR. (b) Increases of $R$ at negative bias are because (b) the defect has moved from A to $\mathrm{B}$ correlated with Vo moving away from CFR. $5 \mathrm{~nm} \mathrm{HfO}_{2}$, HRS.

\section{during $1^{\text {st }}$ test and stay at $\mathrm{B}^{\prime}$ during $2^{\text {nd }}$ test $(\approx B)$.}
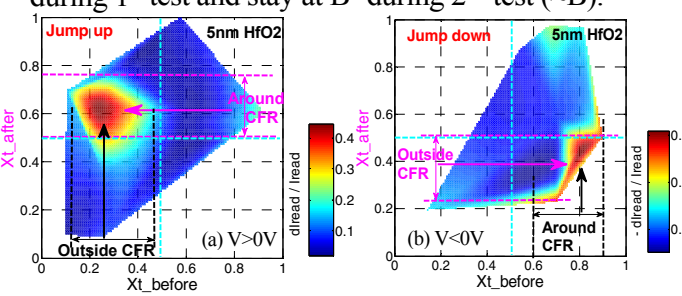

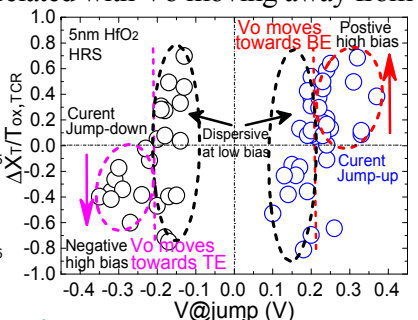

$-1.6 \mathrm{~V}$, Icc $=150 \mathrm{uA}$. (b) $\tau_{\mathrm{c}}$ and $\tau_{\mathrm{e}}$ measured before/after the R jump, from which (c) defect movement in $\left(\mathrm{X}_{\mathrm{T}}, \mathrm{E}_{\mathrm{T}}\right)$ is extracted.

Fig.6 $\Delta$ Iread/Iread vs $\mathrm{X}_{\mathrm{T}}$ before and after Vo movement. Fig.7 For current jump-ups at V>0.2V, Fig.8 (a) 200 defects are detected at HRS during normal DC (a) The largest Iread increase occurs when Vo moving Vo moves towards BE $\left(\Delta \mathrm{X}_{\mathrm{T}}>0\right)$, and switching on/off cycles obtained in several devices using the towards CFR ( $2^{\text {nd }}$ quadrant). (b) the largest Iread decrease for current jump-downs at $\mathrm{V}<-0.2 \mathrm{~V}$, Vo conditions in Fig.3, which clearly shows the CFR near BE. happens when Vo moves out of CFR ( $4^{\text {th }}$ quadrant). No moves towards TE $\left(\Delta \mathrm{X}_{\mathrm{T}}<0\right)$. Vo moves (b) Contour plot of detected local defect numbers confirms jumps are observed in the blank (white) region. HRS. in either direction at $-0.2 \mathrm{~V}<\mathrm{V}<0.2 \mathrm{~V}$.

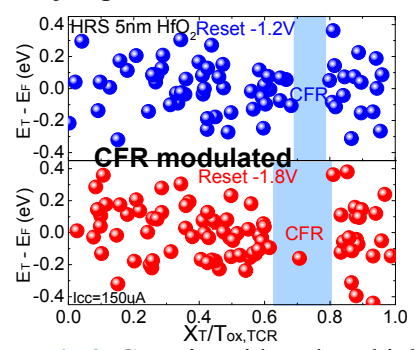

Fig.9 CFR is widened at higher Vreset, and $\mathrm{R}$ at HRS increases from $12.5 \mathrm{k} \Omega$ to $50 \mathrm{k} \Omega$. Vset=1V

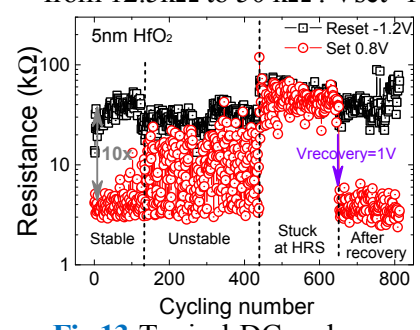

Fig.13 Typical DC endurance test results, where cycling underwent four phases: stable, unstable, stuck at HRS failure, and after recovery.

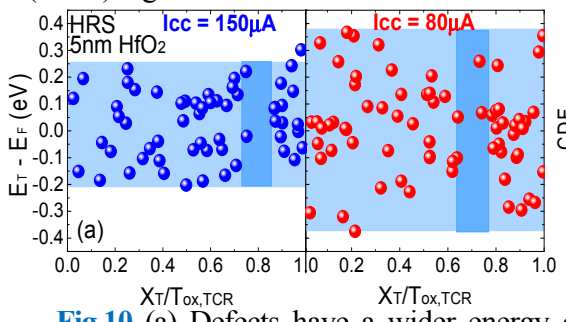

Fig.10 (a) Defects have a wider energy distribution at a

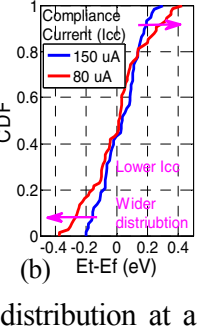

that the least defects are detected at CFR. Note this defect profile does not provide information on actual defect density.
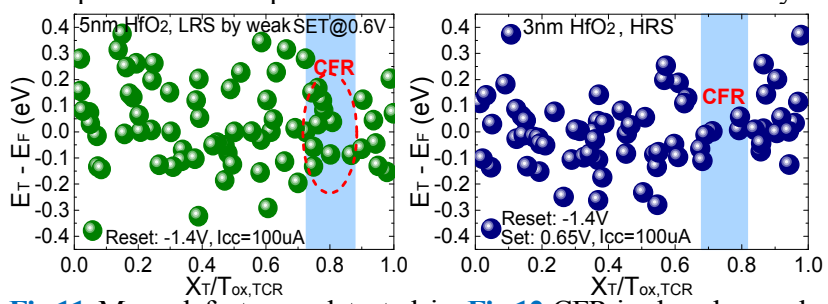

lower compliance ICC, probably due to weaker regulation Fig.11 More defects are detected in Fig.12 CFR is also observed during SET. (b) CDF plot of defect energy distribution. CFR at LRS after a relatively weaker in devices with $3 \mathrm{~nm}^{\mathrm{HfO}} 2$ Vset $=1 \mathrm{~V}$, Vreset $=-1.6 \mathrm{~V}$
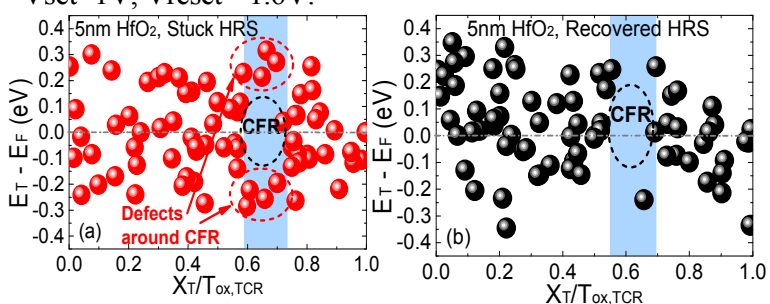

SET (e-tunnelling still dominates)

layer near the BE.

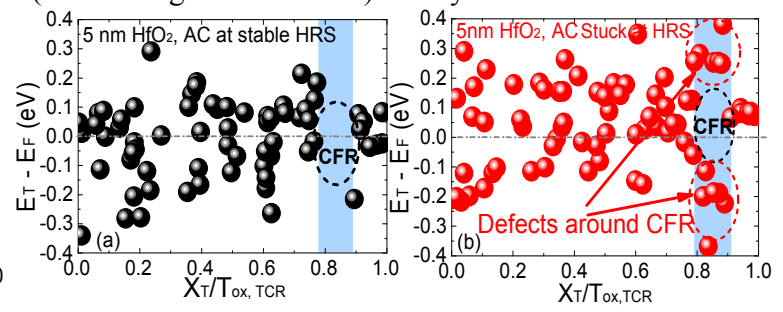

Fig.14 Defects detected during cycling. (a) Stuck at HRS failure Fig.15 Similar failure mode has also been observed under phase. CFR after the failure is surrounded by defects at lower or AC operations, where (a) defects surrounding CFR higher energy levels, causing SET failure. (b) After recovery, the cannot be observed during normal/stable cycles, (b) but defect profile also recovered, providing strong support for the can be observed in failed device stuck at HRS. role of defects surrounding CFR causing device SET failure. $\mathrm{t}_{\text {set/reset }}=20 \mathrm{~ns}, \mathrm{~V}_{\text {set }}=1.4 \mathrm{~V}, \mathrm{~V}_{\text {reset }}=-2 \mathrm{~V}$. 